\title{
STUDY OF THE OPTIMAL HARVESTING CONTROL FOR AN ELLIPTIC PROBLEM WITH SLOW DIFFUSION
}

\begin{abstract}
A. Suárez
To Professor Luis Adauto Medeiros on the occasion of his eightieth birthday

Abstract

In this paper our aim is to present a survey of known results of an optimal control problem with concave non-quadratic cost functional and a state equation arising from population dynamics. First, we study in detail the state equation, and then we show existence and uniqueness of optimal control and also a numerical approximation of the optimal control.
\end{abstract}

\section{Introduction}

Consider a regular domain $\Omega$ of $\mathbb{R}^{N}, N \geq 1$, and the following payoff functional

$$
J: L_{+}^{\infty}(\Omega) \mapsto \mathbb{R}, \quad J(f):=M \int_{\Omega} f u_{f}-K \int_{\Omega} f^{2}
$$

where $L_{+}^{\infty}(\Omega):=\left\{f \in L^{\infty}(\Omega): f(x) \geq 0\right.$ a. a. $\left.x \in \Omega\right\}, M$ and $K$ are positive constants and $u_{f}$ is the maximal solution (see Section 2) of the state equation, the degenerate or slow diffusion logistic equation

$$
\begin{cases}-\Delta u=(a-f) u^{\alpha}-b u^{\beta} & \text { in } \Omega, \\ u=0 & \text { on } \partial \Omega .\end{cases}
$$

The author thanks to the Spanish Ministry of Science and Technology under grant BFM2003-06446.

Key Words. Degenerate logistic equation, optimal control, optimality system. AMS Classification. 49J20, 49K20, 35J65, 92D25. 
Throughout this paper $a, f$ and $b$ are bounded functions with $a$ strictly positive, $b$ non-negative and nontrivial, $a-f$ can change sign and $\alpha$ and $\beta$ satisfy

$$
0<\alpha<1, \quad \alpha<\beta .
$$

The solutions of (1.1) can be regarded as the steady states solutions of the corresponding time dependent model. In such case, $u(x)$ stands for the population density and $\Omega$ for the inhabiting area. Since the population is subject to homogeneous Dirichlet boundary conditions, we are assuming that the environment surrounded $\Omega$ is lethal. In such model, $a(x)$ represents the growth rate of the species and the positive function $b(x)$ describes the intro-specific pressure of the species: in the region where $b \equiv 0$ the species is free of this pressure and so $u$ grows according to the Malthus law, while in its complement $u$ grows according to a logistic or Verhulst law. The function $f(x)$, will be considered non-negative, denotes the distribution of control harvesting of the species by reducing the growth rate.

With respect to the functional, $J$ represents the difference between economic revenue measured by $\int_{\Omega} f u_{f}$ and the control cost measured by $\int_{\Omega} f^{2}$. The constants $M$ and $K$ denote the price of the species and the cost of the control, respectively.

Our objective is to know if it is possible to maximize the benefit, that is, mathematically we want to show:

a) The existence of an optimal control, i. e., to find a function $f_{0} \in L_{+}^{\infty}(\Omega)$ such that

$$
J\left(f_{0}\right)=\sup _{f \in L_{+}^{\infty}(\Omega)} J(f) .
$$

b) Under what conditions is this optimal control unique? 
c) Approximate numerically the optimal control.

In population dynamics, it seems that the first work in optimal control is [16], where the case $\alpha=1, b$ strictly positive and homogeneous Neumann boundary conditions is considered. In such work, a bounded set of controls is considered, the existence of optimal control is shown, as well as the associated optimality system is obtained. Finally, some computational experiments are made. After that, and always in the case $\alpha=1$ and $b$ strictly positive, in [9], [2] and [18], and under homogeneous Dirichelt boundary conditions and control space $L_{+}^{\infty}(\Omega)$, the existence and uniqueness of optimal control are proved. Also, via optimality system, the optimal control is numerically approximated.

However, the case $\alpha<1$ has not been considered previously in our view (see [20] for a related problem). Being $\alpha<1$, some important technical difficulties appear, and over all, a drastic change in the results on the state equation. Indeed, for the case $\alpha=1$ any non-negative and nontrivial solution is in fact positive; however in the case $\alpha<1$ solutions $u \geq 0, u \neq 0$ and no positive in the whole domain exist.

First, we enunciate the main results concerning to the state equation. Then, our aim will be to summary the papers [4] and [5] where the optimal control problem in the case $\alpha<1$ was studied. We show the existence of optimal control, and for $\lambda$ small the uniqueness of optimal control and a characterization of the optimal control via the optimality system is also derived, which further leads to approximating the optimal control. 


\section{State equation}

Equation (1.1) includes the following one (under the change of variable $w^{m}=u$ )

$$
\begin{cases}-\Delta w^{m}=(a-f) w-b w^{2} & \text { in } \Omega, \\ w=0 & \text { on } \partial \Omega\end{cases}
$$

which was introduced in population dynamics by Gurtin and MacCamy in [10] for describing the dynamics of biological populations whose mobility depends upon their density. In this context, $m>1$ (nonlinear slow diffusion) means that the diffusion is slower than in the linear case $m=1$, which gives rise to more realistic biological results, see [10].

By biological meaning, we are only looking for non-negative and nontrivial solutions. Moreover, by elliptic regularity, any bounded solution of (1.1) belongs to $C^{1, \alpha}(\bar{\Omega})$.

We first recall the case $m=1$ in (2.1) (or equivalently $\alpha=1$ in (1.1)). We need previously some notation: given a regular domain $D \subset \mathbb{R}^{N}$ and a function $g \in L^{\infty}(D)$, we denote by $\lambda_{1}^{D}(-\Delta+g)$ the first eigenvalue of the problem

$$
-\Delta u+g(x) u=\lambda u \text { in } D, \quad u=0 \text { on } D \text {. }
$$

It holds (see Theorem 3.5 in [8]):

Proposition 2.1. Assume $\alpha=1$ and that $B_{0}:=\operatorname{int}\{x \in \Omega: b(x)=0\}$ is regular.

a) Any solution $u \geq 0, u \neq 0$ of (1.1) is in fact $u>0$ in the whole domain $\Omega$.

b) There exists a positive solution of (1.1) if, and only if,

$$
\lambda_{1}^{\Omega}(-\Delta+f-a)<0<\lambda_{1}^{B_{0}}(-\Delta+f-a) .
$$


When there exists, the solution is unique and globally stable.

Observe that either the solution is trivial $u \equiv 0$ or it is positive in all the domain. On the other hand, the existence of solution depends on a global condition, specifically of the behavior of $a-f$ in a determined domain. Moreover, in case of existence, there is uniqueness. Finally, we would like to recall that if $\lambda_{1}^{B_{0}}(-\Delta+f-a) \leq 0$ the solutions corresponding to the associated parabolic problem are not bounded, see [8].

A drastic change occurs when $m>1$, due to that strong maximum principle does not hold in general, appearing solutions $u \geq 0, u \neq 0$ and not strictly positive, that is the set

$$
\Omega_{0}:=\{x \in \Omega: u(x)=0\} \neq \emptyset,
$$

this set is called dead core. Moreover, as we can see in the following result, all the solutions are bounded and the multiplicity of solutions appears; as well as the condition assuring the existence of solution is local. Given $g \in L^{\infty}(\Omega)$ we denote by

$$
g_{M}:=\operatorname{ess} \sup _{\Omega} g \quad g_{L}:=\operatorname{ess} \inf _{\Omega} g .
$$

We can summarize the main result as follows:

Theorem 2.2. $\quad$ a) There exists a maximal solution of (1.1), denoted by $u_{f} \geq 0$. Moreover, the map $f \mapsto u_{f}$ is continuous and decreasing, and so $\left\|u_{f}\right\|_{\infty} \leq\left\|u_{0}\right\|_{\infty} \leq \mathcal{K}, \quad$ for some $\mathcal{K}$ independent of $f$

b) The maximal solution $u_{f} \geq 0$, it is not trivial if, and only if, $(a-f)_{M}>0$.

c) If $(a-f)_{L}>0$ then $u_{f}>0$ and it is the unique positive solution of (1.1). Moreover, if there exists a positive solution, this is the unique positive solution. 
d) We can choose functions $f$ such that $u_{f}$ has dead core.

e) If the dead core of $u_{f}$ has $k$ connected components $(k \geq 1)$, then there exist exactly $2^{k}-1$ solutions of (1.1).

Equation (1.1) was previously studied in [11] and [19], where basically paragraph e) and fist part of a) were proved. The rest of results have been proved in [6] and [7].

\section{Optimal control}

Rescaling the functional $J$, we can consider

$$
J: L_{+}^{\infty}(\Omega) \mapsto \mathbb{R}, \quad J(f):=\int_{\Omega} \lambda f u_{f}-f^{2},
$$

with $\lambda>0$. First we can prove the following result, which provides us a bound for any optimal controls and it shows that at the optimal control the corresponding solution $u_{f}$ has not dead core:

Lemma 3.1. Let $f$ be an optimal control. Then,

$$
f \leq \lambda \mathcal{K}
$$

Moreover, $u_{f}>0$ in $\Omega$.

With respect to the existence of optimal control, thank to (3.1) we can take a maximizing sequence and pass to the limit, obtaining

Theorem 3.2. There exists an optimal control, $i$. e., $f \in L_{+}^{\infty}(\Omega)$ such that

$$
J(f)=\sup _{g \in L_{+}^{\infty}(\Omega)} J(g) .
$$

Moreover, the benefit is positive, i.e., $\sup _{g \in L_{+}^{\infty}(\Omega)} J(g)>0$. 
In order to obtain the uniqueness result, we will use two different ways. First, again thank to the bound (3.1), we have that the set of optimal controls belongs to a convex, specifically to the set

$$
I:=[0, \lambda \mathcal{K}]
$$

Hence, if we show that the functional $J$ is concave, we will deduce the uniqueness of optimal control. For that, we calculate the derivative of $J$ in the following set:

$$
\mathcal{C}:=\left\{f \in L^{\infty}(\Omega):(a-f)_{L}>0\right\} .
$$

Proposition 3.3. $J$ is Fréchet differentiable and

$$
J^{\prime}(f)(g)=\int_{\Omega}\left(\lambda u_{f}-\lambda u_{f}^{\alpha} P_{f}-2 f\right) g, \quad \forall f \in \mathcal{C}, \forall g \in L^{\infty}(\Omega),
$$

where for any $f \in \mathcal{C}, P_{f} \in C_{0}^{1}(\bar{\Omega})$ is the unique solution of

$$
\begin{cases}-\Delta P_{f}+M_{f}(x) P_{f}=f & \text { in } \Omega, \\ P_{f}=0 & \text { on } \partial \Omega,\end{cases}
$$

being

$$
M_{f}=-\alpha(a-f) u_{f}^{\alpha-1}+\beta b u_{f}^{\beta-1} .
$$

Some remarks are in order: observe that (3.3) is a linear elliptic equation but with a potential $M_{f}$ blowing up at the boundary of $\Omega$. In spite of that, we have solved this difficulty because $M_{f}$ blows up in a controlled way, due to that $f \in \mathcal{C}$. Indeed, since $f \in \mathcal{C}$ it follows by Theorem 2.2 that $u_{f}>0$ and in fact $u_{f}$ belongs to the interior of the positive cone of $C_{0}^{1}(\bar{\Omega})$. This allows us to solve (3.3), see for instance Theorem 2.5 in [5].

On the other hand, having the derivative of $J$ only in $\mathcal{C}$ is not a restriction, because thank to (3.1) there exists $\lambda_{0}>0$ such that for $\lambda<\lambda_{0}$ it holds that

$$
f \in \mathcal{C} .
$$


The next result is essential in our work, it is a complex technical result (Theorem 4.6 in [5]):

Theorem 3.4. There exists $\Lambda>0$ such that for $0<\lambda<\Lambda$ the maps

$$
f \in[0, \lambda \mathcal{K}] \mapsto u_{f}, P_{f}, u_{f}^{\alpha} P_{f}
$$

are Lipschitz continuous, with the Lipschitz constants independent of $\lambda$, in the following sense

$$
\max \left\{\left\|u_{f}-u_{g}\right\|_{\infty},\left\|P_{f}-P_{g}\right\|_{\infty},\left\|u_{f}^{\alpha} P_{f}-u_{g}^{\alpha} P_{g}\right\|_{\infty}\right\} \leq L\|f-g\|_{2} .
$$

We are ready to show:

Theorem 3.5. There exists $\Lambda_{0}>0$ such that if $\lambda<\Lambda_{0}$, there exists an unique optimal control.

Idea of the proof: Take $\lambda<\lambda_{0}$ of (3.4), and so $f \in \mathcal{C}$. Take also $\lambda<\Lambda$ of Theorem 3.4, then using Proposition 3.3 we get

$$
\left(J^{\prime}(f)-J^{\prime}(g)\right)(f-g)=\int_{\Omega}\left[\lambda\left(u_{f}-u_{g}\right)+\lambda\left(u_{g}^{\alpha} P_{g}-u_{f}^{\alpha} P_{f}\right)-2(f-g)\right](f-g) .
$$

Observe that, by the Hölder inequality

$$
\int_{\Omega}\left(u_{f}-u_{g}\right)(f-g) \leq\left(\int_{\Omega}\left(u_{f}-u_{g}\right)^{2}\right)^{1 / 2}\left(\int_{\Omega}(f-g)^{2}\right)^{1 / 2} \leq C L \int_{\Omega}(f-g)^{2} .
$$

where $L$ is the Lipschitz constant of Theorem 3.4 and $C$ a positive constant.

Thus, we deduce

$$
\left(J^{\prime}(f)-J^{\prime}(g)\right)(f-g) \leq \int_{\Omega}(\lambda L C-2)(f-g)^{2}<0
$$

This conclude that $J$ is concave, and the uniqueness follows.

As a consequence of (3.2), we get easily 
Corollary 3.6. Let $f \in L_{+}^{\infty}(\Omega)$ an optimal control. Then for $\lambda<\lambda_{0}$,

$$
f=\frac{1}{2} \lambda\left(u_{f}-u_{f}^{\alpha} P_{f}\right)^{+} .
$$

We can derive now the optimality system, for that we use the above expression and substitute it in (2.1), and the equation (3.3).

Proposition 3.7. There exists $\Lambda_{1}>0$ such that if $\lambda \leq \Lambda_{1}$, then

$$
P_{f} \leq u_{f}^{1-\alpha} .
$$

So, if $f$ is an optimal control, we have

$$
f=\frac{\lambda}{2} u_{f}\left(1-u_{f}^{\alpha-1} P_{f}\right) .
$$

As consequence, any optimal control $f$ can be expressed as in (3.5), where the pair $\left(u_{f}, P_{f}\right):=(u, P)$ satisfies

$$
\begin{cases}-\Delta u=u^{\alpha}\left(a-\frac{\lambda}{2} u+\frac{\lambda}{2} u^{\alpha} P-b u^{\beta-\alpha}\right) & \text { in } \Omega, \\ -\Delta P+\left(-\alpha a u^{\alpha-1}+\beta b u^{\beta-1}\right) P=\frac{\lambda}{2}\left(u-u^{\alpha} P(1+\alpha)+\alpha u^{2 \alpha-1} P^{2}\right) & \text { in } \Omega, \\ u=P=0 & \text { on } \partial \Omega,\end{cases}
$$

and $u>0$.

The former result says that, when $\lambda$ is small enough, if $f$ is an optimal control, then $\left(u_{f}, P_{f}\right)$ is a solution of (3.6). We are going to prove now that, for a range of $\lambda$, there exists a unique positive solution of (3.6) verifying $u^{1-\alpha} \geq P$ and so the unique optimal control will be

$$
f=\frac{\lambda}{2}\left(u-u^{\alpha} P\right) .
$$

Observe that the system (3.6) is nonlinear in the variables $u$ and $P$ with some nonlinearities singular. So, the study of this system is difficult, an even more the uniqueness, that as it is well know is one of the hardest problem in elliptic problems. We can show the following result: 
Theorem 3.8 (Uniqueness of optimal control). There exists $\Lambda_{2}>0$ such that for $\lambda \leq \Lambda_{2}$, (3.6) possesses a unique positive solution $(u, P)$ satisfying $u^{1-\alpha} \geq P$.

Proof: Define the map:

$$
\mathcal{T}: I:=[0, \lambda \mathcal{K}] \subset L_{+}^{\infty}(\Omega) \mapsto L_{+}^{\infty}(\Omega), \quad f \mapsto \mathcal{T}(f)=\frac{\lambda}{2}\left(u_{f}-u_{f}^{\alpha} P_{f}\right)
$$

By Theorem 3.4, for $\lambda<\Lambda, \mathcal{T}$ is a Lipschitz continuous function with a Lipschitz constant of the form $\lambda \mathcal{L} / 2$, where $\mathcal{L}$ is the corresponding one for the function $f \mapsto u_{f}-u_{f}^{\alpha} P_{f}$. So, we can choose $\Lambda_{2}:=\min \left\{\Lambda, \frac{2}{\mathcal{L}}\right\}$ such that for $\lambda \leq \Lambda_{2}, \mathcal{T}$ is a contractive function.

Assume that there exist two positive solutions $\left(u_{i}, P_{i}\right), i=1,2$ of (3.6) with $u_{i}^{1-\alpha} \geq P_{i}$. We define

$$
f_{i}=\frac{\lambda}{2}\left(u_{i}-u_{i}^{\alpha} P_{i}\right) \in I, \quad i=1,2
$$

Hence, by (3.6) and the uniqueness of $u_{f}$ (see Theorem $2.2 \mathrm{e}$ )), we have that

$$
u_{i}=u_{f_{i}}, \quad P_{i}=P_{f_{i}}, \quad \Rightarrow \mathcal{T}\left(f_{i}\right)=f_{i} \quad i=1,2 .
$$

Since $\mathcal{T}$ is contractive, it follows that $f_{1}=f_{2}$, and again by the uniqueness of $u_{f}$ we have that $u_{f_{1}}=u_{f_{2}}$, hence $u_{1}=u_{2}$, and so $P_{1}=P_{2}$. This completes the proof.

Now, we want to approximate the optimal control, for which we approximate the solutions of (3.6). We are going to use the sub-supersolution method, which provides not only the existence of solution, but sequences approaching the solutions. 
We can define (see [5]) a function $B(x, u, p)$ increasing in $p$, and, depending on the values of $\alpha$ and $\beta$, functions $C(x, u, p)$ decreasing in $u$ and $D(x, u, p)$ increasing in $u$ such that the optimality system (3.6) can be written as

$$
\begin{cases}-\Delta u=B(x, u, p) & \text { in } \Omega, \\ -\Delta p=C(x, u, p)+D(x, u, p) & \text { in } \Omega, \\ u=p=0 & \text { on } \partial \Omega .\end{cases}
$$

Now, we are going to apply the sub-supersolution method to this system and obtain

Proposition 3.9. There exists a pair $(\underline{u}, \bar{u})-(\underline{p}, \bar{p})$ of subsupersolution of the above system, and so there exist sequences

$$
\begin{gathered}
\underline{u}=u_{1} \leq u_{2} \leq \ldots \leq u_{n} \leq u^{n} \leq u^{n-1} \leq \ldots \leq u^{1}=\bar{u} \\
\underline{p}=p_{1} \leq p_{2} \leq \ldots \leq p_{n} \leq p^{n} \leq p^{n-1} \leq \ldots \leq p^{1}=\bar{p}
\end{gathered}
$$

and

$$
u_{n} \nearrow u_{*}, u^{n} \searrow u^{*}, p_{n} \nearrow p_{*}, p^{n} \searrow p^{*}
$$

and for any solution $(u, p)$ of (3.6), we get

$$
u_{n} \leq u_{*} \leq u \leq u^{*} \leq u^{n}, \quad p_{n} \leq p_{*} \leq p \leq p^{*} \leq p^{n}
$$

and $\left(u_{*}, u^{*}, p_{*}, p^{*}\right)$ verifies the following system

$$
\begin{cases}-\Delta u_{*}=B\left(x, u_{*}, p_{*}\right) & \text { in } \Omega, \\ -\Delta u^{*}=B\left(x, u^{*}, p^{*}\right) & \text { in } \Omega, \\ -\Delta p_{*}=C\left(x, u^{*}, p_{*}\right)+D\left(x, u_{*}, p_{*}\right) & \text { in } \Omega, \\ -\Delta p^{*}=C\left(x, u_{*}, p^{*}\right)+D\left(x, u^{*}, p^{*}\right) & \text { in } \Omega, \\ u_{*}=u^{*}=p_{*}=p^{*}=0, & \text { on } \partial \Omega .\end{cases}
$$


Clearly, if $(u, p)$ is the solution of the optimality system (3.6), then $(u, u, p, p)$ is a solution of (3.7). So, to complete the iterative approximation and the convergence of the sequences $\left\{u_{n}\right\},\left\{u^{n}\right\},\left\{p_{n}\right\},\left\{p^{n}\right\}$ to the unique solution, $(u, p)$, of the optimality system, it is sufficient to prove the uniqueness of the solution for the system (3.7).

With a similar argument to the used in Theorem 3.8, we have

Theorem 3.10. There exists a positive constant $\Lambda_{4}$ such that, if $\lambda \leq \Lambda_{4}$, then the system (3.7) possesses a unique solution.

Remark 3.11. a) The existence and uniqueness results are still true for more general functionals as

$$
J(g):=\int_{\Omega}\left(\lambda h(g) u_{g}-k(g)\right),
$$

where $h \in C^{1}\left(\mathbb{R}^{+} ; \mathbb{R}^{+}\right), k \in C^{2}\left(\mathbb{R}^{+} ; \mathbb{R}^{+}\right) ; h(s)=0$ if, and only if, $s=0$, and $k(s)=0$ if, and only if, $s=0 ; h$ is concave and $k$ strictly convex satisfying $k^{\prime \prime}(s) \geq k_{0}>0$ for some $k_{0}$. Moreover:

$$
\text { (H) } \quad \lim _{t \rightarrow 0} \frac{k(t)}{h(t)}=0, \quad \quad \lim _{t \rightarrow+\infty} \frac{k(t)}{h(t)}=+\infty .
$$

b) Different optimal control problems have been analyzed when the state equation is a system, see for instance the works [17], [13], [15], [14], [1], [3] and [12]. In all of them, the control appears in both equations, being an interesting open problem the case with the control acting in only one equation.

\section{References}

[1] Arino, O.; Montero, J. A., Optimal control of a nonlinear elliptic population system, Proc. Edinburgh Math. Soc., 43 (2000), 225-241. 
[2] Cañada, A.; Gámez, J. L.; Montero, J. A., Study of an optimal control problem for diffusive nonlinear elliptic equations of logistic type, SIAM J. Control Optim., 36 (1998), 1171-1189.

[3] Cañada, A.; Magal, P.; Montero, J. A., Optimal control of harvesting in a nonlinear elliptic systems arising fron population dynamics, J. Math. Anal. Appl., 254 (2001), 571-586.

[4] Delgado, M., Montero, J. A. Suárez, A., Optimal control for the degenerate elliptic logistic equation, Appl. Math. Optim., 45 (2002), 325-345.

[5] Delgado, M.; Montero, J. A.; Suárez, A., Study of the optimal harvesting control and the optimality system for an elliptic problem, SIAM J. Control Optim., 42 (2003), 1559-1577.

[6] Delgado, M.; Suárez, A., On the existence of dead cores for degenerate Lotka-Volterra models, Proc. Royal Society of Edin. A, 130 (2000), 743766.

[7] Delgado, M.; Suárez, A., On the structure of the positive solutions of the logistic equation with nonlinear diffusion, J. Math. Anal. Appl., 268 (2002), 200-216.

[8] Fraile, J. A.; Koch-Medina, P.; López-Gómez, J.; Merino, S., Elliptic eigenvalue problems and unbounded continua of positive solutions of a semilinear elliptic equation, J. Diff. Eqns., 127 (1996), 295-319.

[9] Gámez, J. L.; Montero, J. A., Uniqueness of the optimal control for a Lotka-Volterra control problem with a large crowding effect, ESAIM Control Optim. Calc. Var. , 2 (1997), 1-12. 
[10] Gurtin, M. E.; MacCamy, R. C., On the diffusion of biological populations, Math. Biosci., 33 (1977), 35-49.

[11] Hernández, J., Positive solutions for the logistic equation with unbounded limitations, in "Reaction diffusion systems", Lecture Notes in Pure and Applied Mathematics, 194 (1997), 183-197.

[12] Lenhart, S.; Montero, J. A., Optimal control of harvesting in a parabolic system modelling two subpopulations, Math. Models Methods Appl. Sci., 11 (2001), 1129-1141.

[13] Leung, A. W., Optimal harvesting-coefficient control of steady-state preypredator diffusive Volterra-Lotka systems, Appl. Math. Optim., 31 (1995), $219-241$

[14] Leung, A. W., Positive solutions for systems of PDE and optimal control, Non. Anal., 47 (2001) 1345-1356.

[15] Leung, A. W.; Chen, G. S., Optimal control of multigroup neutron fission systems, Appl. Math. Optim., 40 (1999), 39-60.

[16] Leung, G. S.; Stojanovic, S., Optimal control for elliptic Volterra-Lotka type equations, J. Math. Anal. Appl., 173 (1993), 603-619.

[17] He, F.; Leung, G. S.; Stojanovic, S., Periodic optimal control for competing parabolic Volterra-Lotka-type systems, J. Comp. App. Math., 52 (1994), $199-217$

[18] Montero, J. A., A uniqueness result for an optimal control problem on a diffusive elliptic Volterra-Lotka type equation, J. Math. Anal. Appl., 243 (2000), 13-31. 
[19] Pozio, M. A.; Tesei, A., Support properties of solutions for a class of degenerate parabolic problems, Comm. Partial Diff. Eqns., 12 (1987) 47-75.

[20] Stojanovic, S., Modeling and minimization of extinction in Volterra-Lotka type equations with free boundaries, J. Diff. Eqns., 134 (1997), 320-342.

Dpto. de Ecuaciones Diferenciales y Análisis Numérico

Fac. de Matemáticas, Univ. de Sevilla

Calle Tarfia s/n, 41012-Sevilla, Spain

E-mail: suarez@us.es 